DOI: $10.3901 / J M E .2019 .11 .090$

\title{
喷印柔性压力传感器试验研究*
}

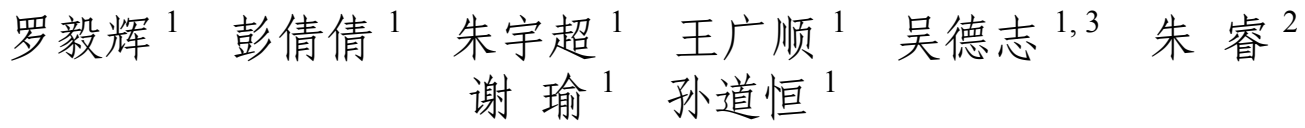

(1. 厦门大学机电工程系 厦门 361005 ;

2. 厦门大学动力工程系 厦门 361005;

3. 厦门大学深圳研究院 深圳 518057)

\begin{abstract}
摘要: 柔性压力传感器是智能机器人和生物医疗等典型应用领域中的关键部件。针对 $10 \sim 50 \mathrm{kPa}$ 中等压力下对柔性压力传感 器的高灵敏度、良好压力分辨率和快速响应需求, 提出在 PDMS 基底上直写喷印石墨烯纳米片(GNPs)/多壁碳纳米管(MWCNT) 构建 $\mathrm{S}$ 型折线图案化敏感单元, 结合封装层微结构阵列, 制备中压高灵敏度、低检测限的柔性压力传感器。试验结果表明, 在压力为 $0 \sim 15 \mathrm{kPa}$ 和 $15 \sim 40 \mathrm{kPa}$ 的条件下, 该传感器灵敏度分别为 $0.114 \mathrm{kPa}^{-1}$ 和 $1.41 \mathrm{kPa}^{-1}$, 响应/恢复速度快(约 $100 \mathrm{~ms} / 50$ $\mathrm{ms}$ )。同时, 其也可检测低至约 $3 \mathrm{~Pa}$ 的微小压力。同时, 该传感器更是能对不同发声进行准确的区分识别, 对不同的指压信 号进行精确稳定反馈。可见, 喷印制造柔性压力传感器将为语音识别、人工假肢、制备高性能电子皮肤和医疗康复器件等提 供可能的优选方案。
\end{abstract}

关键词：直写喷印；柔性压力传感器；石墨烯纳米片；微结构；电子皮肤

中图分类号: TP212

\section{Experimental Study of Flexible Pressure Sensor via Direct Writing}

\section{LUO Yihui $^{1}$ PENG Qianqian ${ }^{1}$ ZHU Yuchao ${ }^{2}$ WANG Guangshun ${ }^{1}$ WU Dezhi ${ }^{1,3}$ ZHU Rui ${ }^{2} \quad$ XIE Yu $^{1}$ SUN Daoheng ${ }^{1}$}

(1. Department of Mechanical \& Electrical Engineering, Xiamen University, Xiamen 361005;

2. Department of Power Engineering, Xiamen University, Xiamen 361005;

3. Shenzhen Research institute of Xiamen University, Shenzhen 518057)

\begin{abstract}
Flexible pressure sensors are crucial building blocks for potential applications including intelligent robots and biomedical engineering. According to its demand of high resistivity, good pressure resolution and fast response for sensing medium pressure between $10 \mathrm{kPa}$ and $50 \mathrm{kPa}$, a flexible pressure sensor is fabricated by direct writing graphene nanoplatelets (GNPs) / multi-walled carbon nanotubes (MWCNT) composite on a PDMS substrate to construct an S-shape sensing element, together with a microstructure-arrayed package component. Experiment results show that its sensitivity is about $0.114 \mathrm{kPa}^{-1}$ and $1.41 \mathrm{kPa}^{-1}$, under pressure of $0-15 \mathrm{kPa}$ and $15-40 \mathrm{kPa}$ respectively. Its response / recovery time is about $100 / 50 \mathrm{~ms}$, which is faster than that of most medium pressure sensors. It also can sense subtle pressure change about $3 \mathrm{~Pa}$. It is characterized to be of better repeatability and cycling stability. At the same time, the different phonation can be accurately distinguished and recognized by the sensor, and the different finger pressure signals can be accurately and stably feedback. Thus, such sensors will offer a possible optimized choice for speech recognition, artificial limb, fabrication of electronic skins and medical rehabilitation devices with high performance.
\end{abstract}

Key words: direct write; flexible pressure sensor; graphene nanoplatelets; microstructures; electronic skins

\section{0 前言}

可共形贴附于结构表面的柔性传感器可真实高

* 国家自然科学基金(91648114)、中央高校基本业务费专项资金(厦门大 学: 20720170037)、浙江省重点研发计划(2018C01036)和深圳市科技计 划项目(20180211151853569)资助项目。20181126 收到初稿, 20190301 收到修改稿

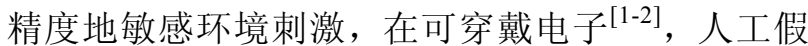
肢 ${ }^{[3-4]}$, 人体健康监测 ${ }^{[5-6]}$ 和机器人 ${ }^{[7-8]}$ 等领域应用广 泛，也是人机交互技术的基础关键部件。《中国制造 2025》和《“十三五”创新规划》等国家、地方政策 中都将智能感知、可穿戴技术列为研究重点。可见， 柔性传感器是未来的发展方向。压力是多种刺激信 号中的重要参数之一，以压阻传感为典型压力敏感 
机制的压力传感器应用最为广泛。传统压阻传感器 的敏感材料一般采用半导体, 导电聚合物, 填充有 导电炭黑颗粒的弹性橡胶和金属/金属氧化物纳米 线等。但这些传统材料大部分的敏感性差, 检测分 辨率低 $(>0.5 \mathrm{kPa})^{[9-10]}$, 不稳定, 限制了其在电子皮 肤上的应用。而以碳纳米管, 石墨烯 ${ }^{[11-13]}$ 等碳系材 料作为敏感材料的新型柔性电子皮肤因具有高灵敏 度和优异的稳定性引起了业界的高度关注。

根据生物皮肤的压力传感特性, 主要压力感应 包括低压感应 $(<10 \mathrm{kPa})$ 和中压感应 $(10 \sim 100$ $\mathrm{kPa})^{[14-15]}$ 。为了更加接近生物皮肤的敏感特性, 提 高柔性压力传感器在低压范围内的敏感特性和响应 速度, 除了引入先进的传感材料外, 构建表面微结 构也是一种可选择的方案。研究报道己证明微结构 或图案化聚二甲基硅氧烷(PDMS)为提高电阻型传 感器的灵敏度提供有效途径。科研人员采用基于 PDMS 制作了许多特殊微结构, 如弹性微金字塔阵 列 ${ }^{[16]}$, 圆顶形结构 ${ }^{[17]}$ 或可逆互锁的纳米纤维微阵列 ${ }^{[18]}$ 。 例如, BAO 等 ${ }^{[19]}$ 使用带新型金字塔结构的 PDMS 薄膜作为介电层来制造柔性压力传感器, 其在 $<2$ $\mathrm{kPa}$ 压力范围内表现出 $0.55 \mathrm{kPa}^{-1}$ 的高灵敏度, 性能 明显高于非结构化薄膜 $\left(0.02 \mathrm{kPa}^{-1}\right)$ 。CHEN 等 ${ }^{[20]}$ 还 制造了一种基于微结构石墨烯薄膜的柔性触觉传感 器, 在 $<100 \mathrm{~Pa}$ 低压范围内具有高灵敏度 $\left(-5.5 \mathrm{kPa}^{-1}\right)$ 。 但这些传感器感测范围小且感测性能很难适应中压 场合 ${ }^{[21-22]}$ 。同时, 这些压力传感器在进行中压检测 时的响应时间较长 $(>0.5 \mathrm{~s})^{[23]}$, 难以适应电子皮肤要 求。同时对于电子皮肤而言, 中压灵敏度和检测限 应至少分别为约 $10 \mathrm{MPa}^{-1}$ 和 $50 \mathrm{~Pa}$ 。所以依然急需 低成本制造技术来构造高灵敏度中等压力传感器中 的可调微结构化表面, 该微结构化表面可用于宽压 力范围及低压检测。因此关于如何制造在中压检测 范围内具有高灵敏度, 快速响应和良好的循环稳定 性柔性电子皮肤压力传感器的挑战依旧是存在的。

同时, 许多文献已经报道了使用碳基纳米材料, 例如石墨烯 $(\mathrm{Gr})$, 氧化石墨烯 $(\mathrm{GO})$ 和碳纳米管 $(\mathrm{CNT})$ 等来制造敏感单元的方法, 包括化学气相沉积 $(C V D)^{[24]}$, 丝网印刷 ${ }^{[25]}$ 和光刻 ${ }^{[20]}$ 等, 但它们一般需 要昂贵的设备并且制造工艺复杂化。因此科学家们 正致力于开发简单, 快速和低成本的方法来制造柔 性压力传感器。直写喷印是制造压力传感器的一种 低成本且快速简单的方法。本文利用直写喷印技术 将石墨烯复合材料图案化沉积在柔性 PDMS 基底上 作为压力敏感单元, 以形成可检测宽域压力的柔性 压力传感器, 利用模具倒模得到具有微结构的 PDMS 封装层, 以提高压力传感器的检测灵敏度和
检测分辨率。试验结果表明, 该传感器具有高灵敏 度, 快速响应, 重复性好, 循环稳定性好等特点。 它在对人体发声和手指触摸动作展示出良好的性能 表明其在医疗健康检测、身体运动和智能假肢等领 域的巨大发展潜力。

\section{1 传感器制备与试验方法}

\section{1 试验材料}

自制石墨烯纳米片混合溶液(石墨烯溶液, Aldrich, MW=12.01 g/mol); 多壁碳纳米管(Aldrich, 碳含量: >98\%); PDMS(Dow Corning Sylgard 184, 基本组分与固化剂质量比为 10:1); 电极材料为 HS-8200 Ag(Hanstars)。

\section{$1.2 \mathrm{GNPs} / \mathrm{MWCNT}$ 溶液前驱体配置}

将多壁碳纳米管按照一定的比例分散在石墨烯 混合液中, 然后将上述溶液使用磁力搅拌器以 200 $\mathrm{r} / \mathrm{min}$ 搅拌 $2 \mathrm{~h}$, 再使用超声波装置(KUDOS, 上海) 在水浴中以 $80 \%$ 功率和 $50 \mathrm{kHz}$ 频率超声 $3 \mathrm{~h}$, 使混 合液充分混合均匀, 避免发生团聚, 保证后续试验 顺利开展。

\section{3 基材制备}

利用搅拌器(JJ-1, 江苏华丰)以 $200 \mathrm{r} / \mathrm{min}$ 速度 摚拌混合聚二甲基硅氧烷(PDMS)后, 将其在 $4{ }^{\circ} \mathrm{C}$ 下脱气 $2 \mathrm{~h}$ 并倒在硅片抛光面上, 经 $300 \mathrm{r} / \mathrm{min}$ 速度 旋涂 $5 \mathrm{~min}$; 接着把 PDMS 置于真空烘箱中抽真空 去气泡处理 $15 \mathrm{~min}$, 最后在 $80{ }^{\circ} \mathrm{C}$ 下保持 $40 \sim 60$ $\mathrm{min}$, 获得厚度约为 $500 \mu \mathrm{m}$ 的 PDMS 基底; 用刀片 将这些膜切成长 $2 \mathrm{~cm}$, 宽 $1 \mathrm{~cm}$ 的长方形; 最后, 通过等离子体(Q150Alpha Plasma, Germany)表面活 化 PDMS 基底 $8 \mathrm{~min}$, 意在提升所喷印 GNPs/ MWCNT 复合材料压力敏感结构与 PDMS 基材表面 的结合力。

\section{4 压力传感器喷印制造}

传感器喷印制造装置采用自制直写喷印系统, 如图 1 所示, 它主要包括自制直写打印模块, 高精

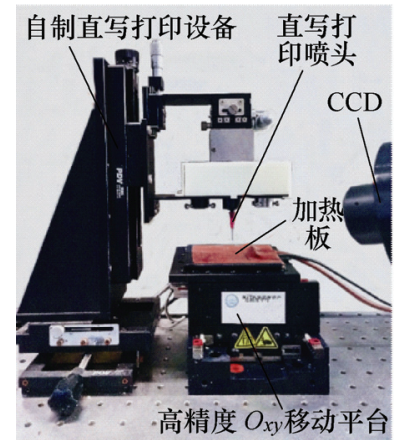

(a) 直写打印设备装置实物图

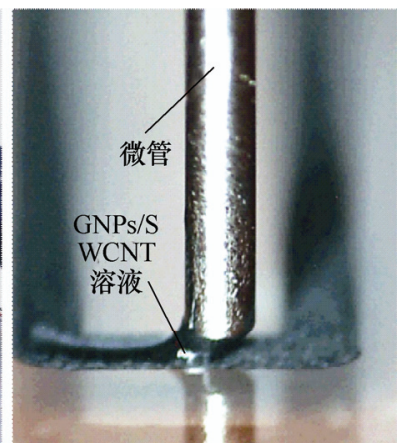

(b) 喷头放大实物图及工作图
图 1 直写喷印系统及其工作图 
度 $O x y$ 运动平台, 平板加热模块和工业相机。计算 机控制 $O x y$ 运动平台实现敏感单元图案化喷印。运 动平台上平板加热模块 $\left(80{ }^{\circ} \mathrm{C}\right)$ 用于直写喷印过程 中促进基材上敏感单元快速固化成型。当喷印开始 后, 溶液被快速输运到针尖(图 1b), 进而喷印沉积 在 PDMS 基材表面, 完成压敏单元图案化喷印制造。

电阻式柔性压力传感器整体制造过程如图 2 所 示: (1) 在直写喷印系统上将 GNPs/MWCNT 混合墨 水根据设定 $\mathrm{S}$ 型折叠图案喷印在 PDMS 基材上, 制 得纳米复合材料均匀分散、输出性能更好的压敏单 元; (2) 将带有图案化敏感单元的 PDMS 基材置于 温度为 $100{ }^{\circ} \mathrm{C}$ 的加热装置上, 在压力敏感单元两端 涂覆导电银浆作为电极, 并引入导线后加热固化;

(3) 利用 3D 打印机打印出具有条纹微结构的模具, 将 PDMS 浇注其上后依然同样采用抽真空、热固化 和裁切, 得到与基材结构尺寸一样的长方形封装层; (4) 将带有微结构的 PDMS 封装层对准覆盖在含敏 感单元的 PDMS 基材上, 热压成型并从玻璃上剥离 后最终制备得带有微结构的双层柔性压力传感器。

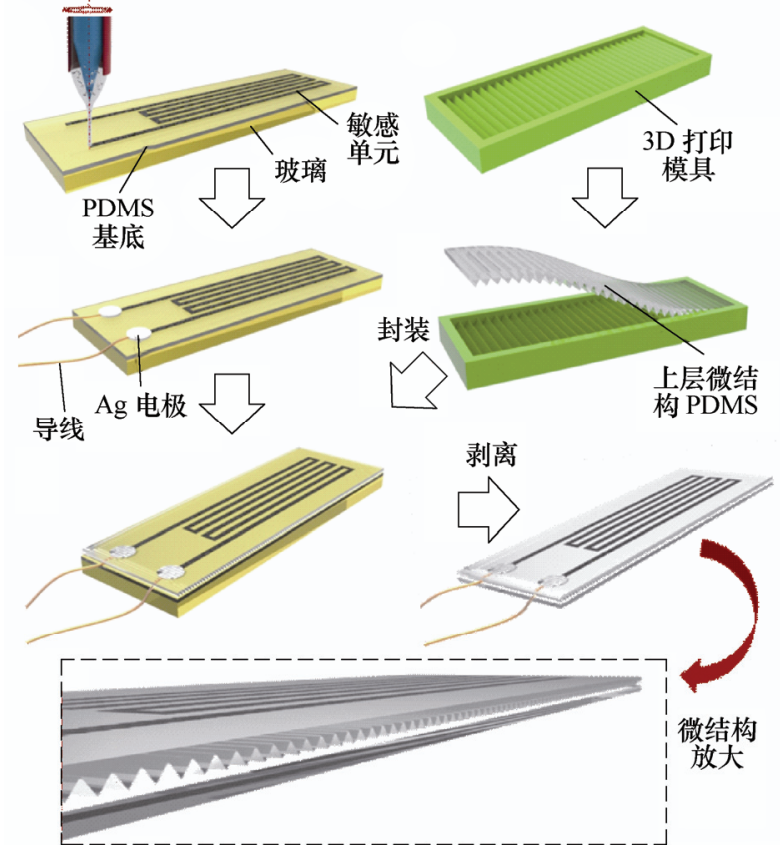

图 2 柔性电阻式压力传感器制造工艺流程

\section{5 表征与测试}

利用连续变倍体视显微镜(SZ66, 中国)及奥特 光学金相显微镜(MIT 系列, 中国)观测敏感单元的 宏观外貌; 敏感单元及 PDMS 微结构封装层采用扫 描电子显微镜(SEM JSM-IT500a, 日本)观测; 从 PDMS 基底上剥离敏感单元样品后, 采用透射电子 显微镜(JEM-1400)考察敏感单元内部微观结构; WITEC CRM200 拉曼系统用于检测样品不同区域 的拉曼光谱; 利用超景深三维显微系统(VHX-5000,
日本)观察敏感单元的立体微观结构特征。

压力传感器的电学特性及压敏特性测试主要通 过压力计 (Handpi HP-5N, 中国)、 $z$ 轴自动运动平台 和数字万用表(Agilent 34410A，美国)等来完成。先 将传感器粘附固定于 $z$ 轴平台的测力计下方, 通过 平台移动, 利用测力计将负载压力施加到柔性压力 传感器上。循环测试是通过设定好施载的频率和加 载遍数后，由 $z$ 轴移动平台进行负载施加。在测试 传感器分辨率时, 先将 $5 \mathrm{~mm} \times 9 \mathrm{~mm}$ 矩形硅片放置 在传感器表面上, 然后再把待测试物放在硅片上, 便于确定压强。

\section{2 传感器材料结构表征}

\section{1 直写喷印墨水}

墨水中 MWCNT 主要为 GNPs 之间的连接提供 更多的导电通路, GNPs 和 MWCNT 的比例对敏感 单元输出性能影响巨大，保证敏感单元的导电性和 压敏性能是制备直写喷印墨水的关键。配置 GNPs/MWCNT 比例分别为 $1: 0,4: 1,1: 1$ 及 $1: 4$ 的墨水用于试验探究。如图 3 所示, 当溶液不含有 MWCNT 时(即比例为 1:0), 尽管 GNPs 表面积大, 但很多 GNPs 之间由于产生阻隔而无法相互接触导 通, 难以形成导电压敏结构; GNPs/MWCNT 比例 为 4:1 时, MWCNT 随机分散在 GNPs 之间, 提供 了有效的导电路径和机械桥接配体，但由于 MWCNT 相对含量较低，只有少部分 GNPs 之间实 现连接, 敏感单元初始电阻可达到 $50 \mathrm{M} \Omega$, 这不 利于后端信号传输。而随着 MWCNT 含量进一步增 加至 1:1 时, MWCNT 为 GNPs 之间的连通提供了 大量连接桥梁, GNPs/MWCNT 之间构成了稳定而

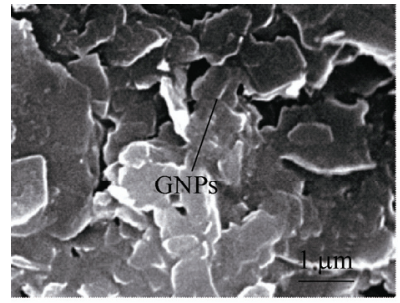

(a) GNPs

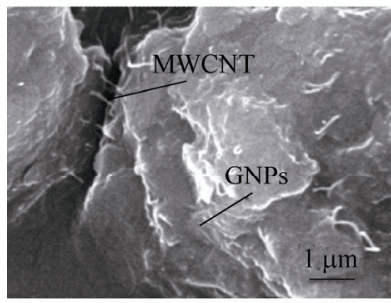

(c) GNPs:MWCNT=1:1

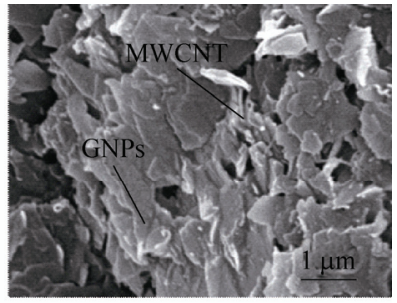

(b) GNPs:MWCNT=4:1

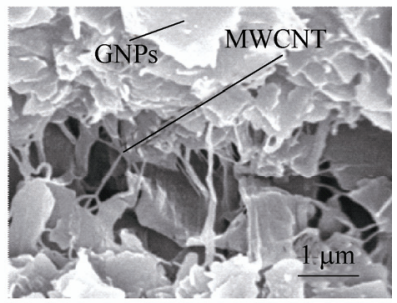

(c) GNPs:MWCNT=1:4
图 $3 \mathrm{GNPs} / \mathrm{MWCNT}$ 不同比例的墨水喷印后 SEM 图 
广泛的 3D 导电网络, 为敏感单元结构提供了更多 的传输通道, 从而增强了敏感单元的导电性和结构 稳定性, 敏感单元初始电阻下降至约 $100 \mathrm{k} \Omega$ 。但当 GNPs/MWCNT 改变为 $1: 4$ 时, 沉积的敏感单元中 MWCNT 含量迅速增加, 为 GNPs 之间连接提供了 过多的连接桥梁, 使得敏感单元电阻进一步下降, 降至约 $15 \mathrm{k} \Omega$, 同时, 导电 $3 \mathrm{D}$ 网络的过多增加使得 敏感单元结构和导电性更加稳定, 在压力作用下的 变化将减小, 这将削弱敏感单元的敏感性能。因此 后续试验中 GNPs 和 MWCNT 的比例设为 $1: 1$ 。

\section{2 直写喷印微结构与基材粘合技术}

直写喷印时以未经任何处理的 PDMS 为基材, 很难在 PDMS 表面沉积形成稳定的图案化结构, 非 常容易出现断裂。这主要是由于 PDMS 表面能原因 导致喷印的墨水难以有效附着在 PDMS 上。即使在 直写过程中已经在 PDMS 上附着上敏感单元, 但随 着固化成型后, 敏感单元或将发生脱落或者趐曲而 无法完全黏附于基底之上(图 4a), 影响敏感单元的 正常功能。为此采用等离子体氧化活化对 PDMS 表 面做处理, 经过处理后, 直写喷印的墨水很容易稳 定地黏附在 PDMS 表面上, 固化成型后, 依旧能在 PDMS 基底表面稳定黏附而不产生脱落, 如图 4b 所示。这是因为等离子体氧化活化使 PDMS 表面产 生活化表面, 活化表面又与活性氧发生化学反应, 使表面层的部分 $-\mathrm{CH}_{3}$ 和 $-\mathrm{CH}_{2}$ 等基团消失, 并嵌入了 一些-OH、- $\mathrm{COOH}$ 等极性基团, 这些极性基团的存 在增加了 PDMS 的表面活性能, 使得 PDMS 表面和 敏感单元牢固地连接在一起 ${ }^{[26]}$ 。

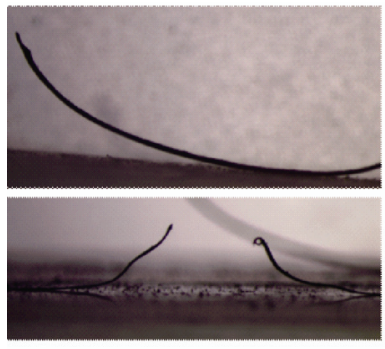
直写的敏感单元趐曲 (a) 未处理 PDMS 基底上

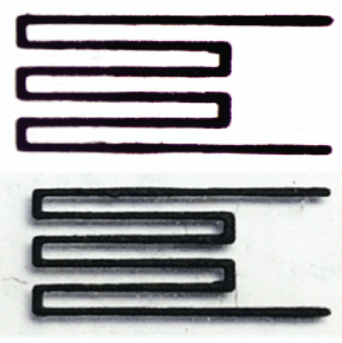

(b) 等离子体氧化活化后 PDMS 基底直写情况
图 4 PDMS 表面直写喷印情况

\section{3 敏感单元结构形貌与性能表征}

直写喷印的柔性压力传感器如图 5a、5b 所示, 基底厚度约 $500 \mu \mathrm{m}$, 长宽为 $20 \times 10 \mu \mathrm{mm}$, 与物体表 面贴附性好。传感器内部敏感单元为 $\mathrm{S}$ 型三折半间 距约 $1 \mathrm{~mm}$ 的折叠结构, 且线长度约 $12 \mathrm{~mm}$ 和 $8 \mathrm{~mm}$ 。 图 $5 \mathrm{c}$ 显示敏感单元的底部线宽约为 $230 \mu \mathrm{m}$, 且其 微观表面凹凸不平, 不规则, 这有利于传感器的灵 敏度提升 ${ }^{[27]}$; 同时横截面为多次往返喷印墨水不断
堆叠滑落呈梯形(图 5d), 高度达到约 $60 \mu \mathrm{m}$, 这 也有利于敏感单元压敏特性的增强, 产生稳定变 形 ${ }^{[28]}$ 。透射电镜图(图 5e)清晰地显示了 GNPs 和 MWCNT 在敏感单元中的良好分散, MWCNT 位于 GNPs 之间并桥接相邻的 GNPs, 构建起了敏感单元 的良好三维导电网络。这就保证了直写喷印得到的 敏感单元中 GNPs/MWCNT 具有良好的分散性和均 匀性, 避免团聚现象的出现。通过拉曼测试(图 5f) 后发现, GNPs/MWCNT 复合材料在约 $1350 \mathrm{~cm}^{-1}$, $1580 \mathrm{~cm}^{-1}$ 和 $2695 \mathrm{~cm}^{-1}$ 处存在三个峰, 对应于 $\mathrm{D}$, $\mathrm{G}$ 和 $2 \mathrm{D}$ 振动模式。2D 峰与石墨烯层数和多层石墨 烯材料的堆叠顺序密切相关, 而这里显示出蓝移和 展宽是由于材料产生多层卷曲引起的 ${ }^{[29]}$ 。复合材料 $\mathrm{D}$ 峰与 $\mathrm{G}$ 峰强度比 $I_{\mathrm{D}} / I_{\mathrm{G}}$ 小于 0.085 , 表明石墨烯在 直写喷印过程中没有显著改变, 没有引入缺陷。同 时 2D 峰显示出一定的不对称性, 一定程度上表明 敏感单元材料呈三维有序性 ${ }^{[30]}$ 。

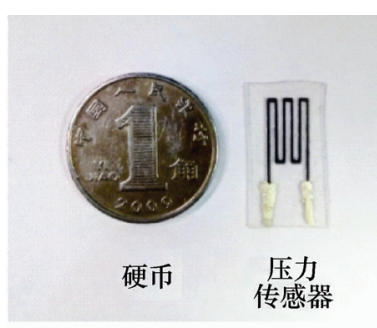

(a) 柔性传感器实物图

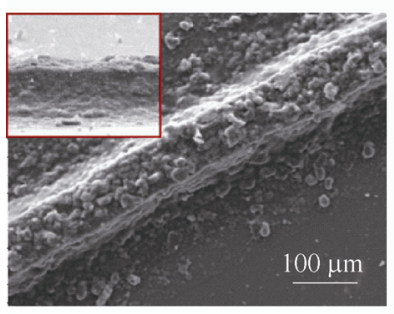

(c) SEM 局部俯视图

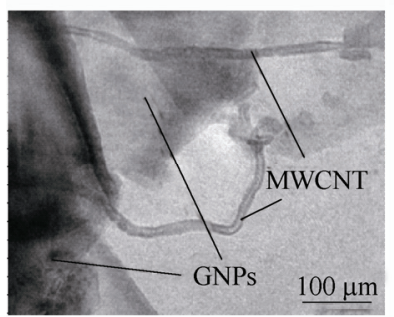

(e) TEM

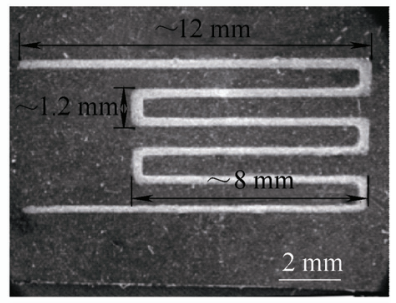

(b) 敏感单元 SEM

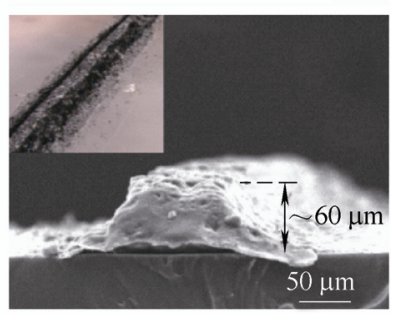

(d) 敏感单元横截面图

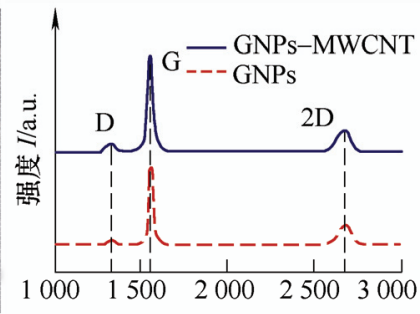

波数 $\mathrm{k} / \mathrm{cm}^{-1}$

(f) 拉曼测试
图 5 敏感单元的微观结构图及其表征

\section{4 带微结构的 PDMS 封装层}

如图 6 所示, 3D 打印模具倒模制造的 PDMS 封装层下表面分布着一排周期性平行线形微结构, 横截面为锥形。相邻峰间距约为 $800 \mu \mathrm{m}$ (图 6a、6b), 单峰高度约为 $320 \mu \mathrm{m}$ (图 6c、6d)。这些具有大量尖 峰的图案化微结构为后续压力传感提供了许多有效 
的接触位置, 为提高敏感单元的灵敏度起到了关键 性作用。

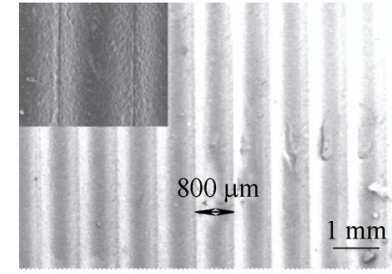

(a) 封装层微结构俯视图

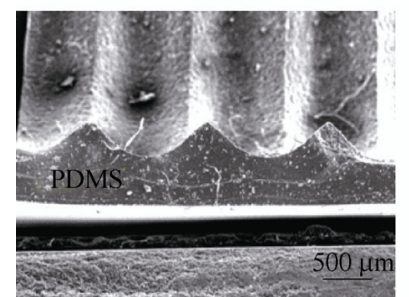

(c) 封装层微结构截面

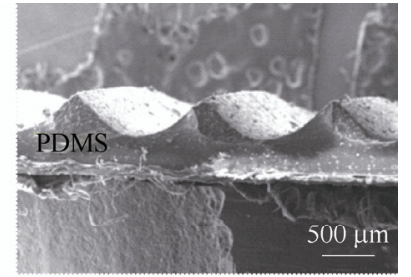

(b) 封装层微结构斜视图

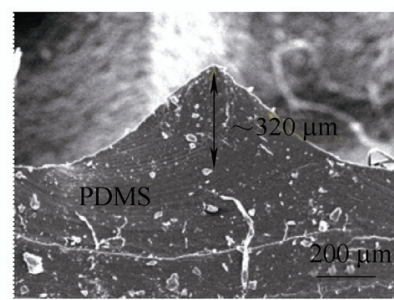

(d) 截面放大
图 6 封装层 PDMS 微结构

\section{3 传感器敏感机理及测试分析}

该柔性压力传感器的工作原理如图 7 所示, 在 没有压力施加的情况下, 敏感单元保持在原来的形 态, GNPs/MWCNT 构成的导电网络具备良好的导 电能力, 电阻不发生变化, 但是当压力增加时敏感 单元大量部位受到上层微结构的压力开始发生形 变, 同时随着压力的不断增大形变也在不断增大, 当敏感单元受到形变影响时, 内部 GNPs/MWCNT 的导电网络开始产生可恢复性破坏, GNPs/MWCNT 之间的导电网络由于形变而张大, 甚至导致导电网 络的断开, GNPs/MWCNT 之间的接触随着形变的 增大变得更加困难, 这也就导致了电阻值随着压力 的增加而不断增大 ${ }^{[31]}$ 。

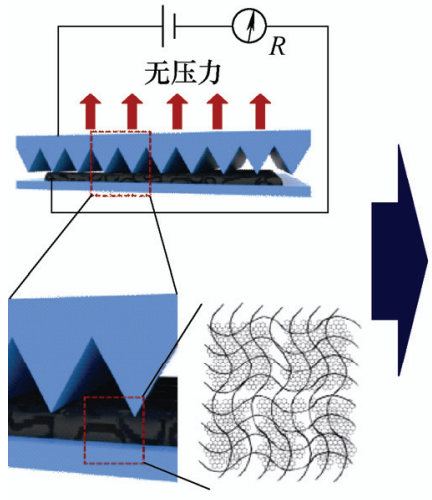

(a) 施压前 (b) 施压后

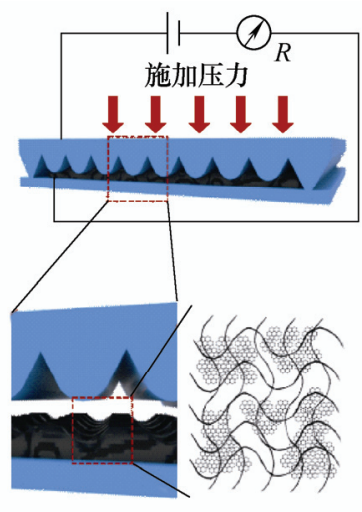

图 7 传感器工作原理图

压力传感器灵敏度系数 $S$ 定义为

$$
\begin{gathered}
S=\frac{\delta\left(\frac{\Delta R}{R}\right)}{\delta p} \\
\Delta R=R_{\mathrm{a}}-R
\end{gathered}
$$

式中, $R_{\mathrm{a}}$ 代表施加压力后敏感单元电阻值, $R$ 代表 未施加压时初始电阻值, $p$ 代表压力值。如图 $8 \mathrm{a}$ 所示, 当封装层表面平滑, 没有微结构时, 传感器 在 $0 \sim 20 \mathrm{kPa}$ 和 $20 \sim 40 \mathrm{kPa}$ 压力范围内的灵敏度分 别达到约 $0.015 \mathrm{kPa}^{-1}$ 和 $0.188 \mathrm{kPa}^{-1}$ 。而引入了平行 阵列微结构后, 在 $0 \sim 15 \mathrm{kPa}$ 和 $10 \sim 40 \mathrm{kPa}$ 压力范 围内的灵敏度提高到 $0.114 \mathrm{kPa}^{-1}$ 和 $1.41 \mathrm{kPa}^{-1}$, 即 灵敏度提升了 8 倍左右。这是由于传感器封装层引 入了微结构, 微结构尖端区域小, 压强增大, 势必 增强了敏感单元中微纳米结构的接触, 同时微结 构的加入，使得敏感单元发生形变的部位也随之 增加, 随着压力不断加大, 敏感单元更多部位的 形变将变得更加剧烈, 这也将引起电阻的变化不 断加剧。

进一步试验探讨柔性压力传感器对外部压力的 响应和回复时间, 得到传感器的压力响应特性。图 $8 \mathrm{~b}$ 显示了在约 $17 \mathrm{kPa}$ 压力加载和卸载下瞬时的电 阻变化, 测试得到的实时阶跃响应曲线反映了柔性 压力传感器的响应特性, 表明传感器具有出色的响 应/回复时间, 对压力存在明显且稳定的响应。随着 外界压力的施加, 电阻几乎立即响应于外部压力, 一旦外部压力消失, 立即恢复到初始值, 在加载和 卸载外部压力的情况下, 响应/回复时间分别达到了 约 $100 \mathrm{~ms}$ 和 $50 \mathrm{~ms}$ 。压力传感器的这种快速响应特 性与其他文献中中压压力传感器的类似 ${ }^{[32]}$ 。为考察 传感器的重复性、可靠性和稳定性在传感器上重复 施加一系列循环压力 $(6 \mathrm{kPa}, 12 \mathrm{kPa}, 18 \mathrm{kPa}, 24 \mathrm{kPa})$ 和进行同一载荷的不断循环测试。如图 $8 \mathrm{c}$ 所示, 当 外部压力从 $6 \mathrm{kPa}$ 增加到 $21 \mathrm{kPa}$ 时, $\Delta R / R$ 相应的从 约 0.48 增加到约 10.88 , 其不仅在施加压力时能在 一个电阻值附近稳定，同时在几个循环内保持很好 的一致性, 表明传感器在中压范围内具有精确的压 力感知能力。如图 $8 \mathrm{~d} 、 8 \mathrm{e}$ 所示, 重复施加约 $15 \mathrm{kPa}$ 的载荷, 进行 1000 次的循环测试, 通过观察, 我 们发现, $\Delta R / R$ 随着压力的增加由 0 变化到约 1.7 , 随着压力的减小又几乎回到初始值的状态, 每一次 $\Delta R / R$ 的振幅和波形都保持了很好的一致性，同时每 一次的响应变化误差都保证在 $1 \%$ 的范围内, 这进 一步表明了该柔性压力传感器具有优异的可靠性和 稳定性，为其在电子皮肤、可穿戴设备压力检测等 领域的应用奠定良好的基础。 

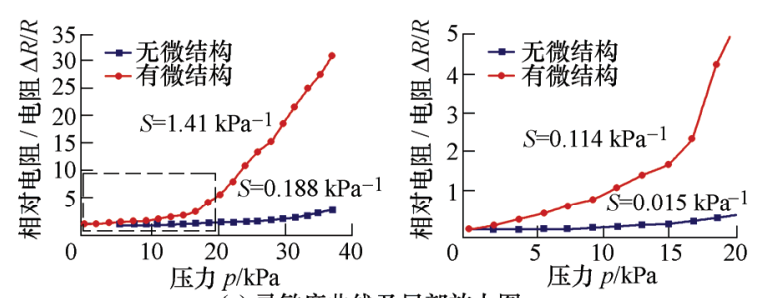

(a) 灵敏度曲线及局部放大图

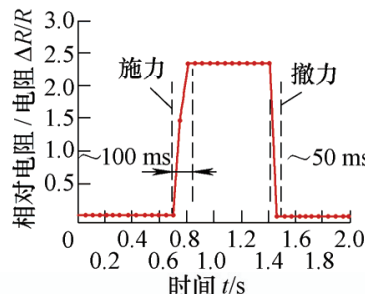

(b) 响应时间

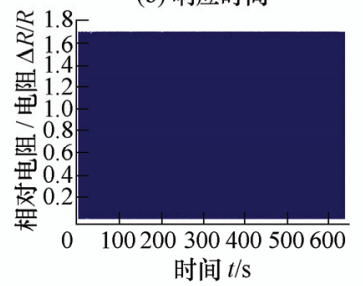

(d) 1000 次循环测试

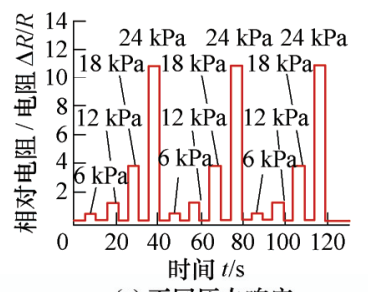

(c) 不同压力响应

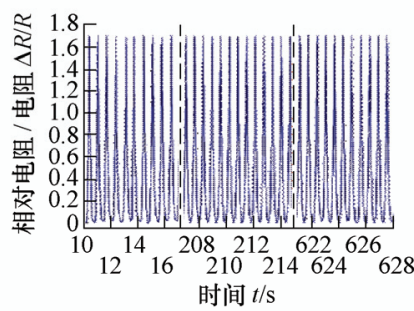

(e) 循环测试截取放大
图 8 压力传感器测试曲线图

分辨率是柔性传感器的一个重要指标, 为此将 一颗米粒(约 $15.8 \mathrm{mg}$ )、一颗绿豆(约 $52.5 \mathrm{mg}$ )或一颗 红豆(约 $156.8 \mathrm{mg}$ ) 放置在传感器上方后移开进行小 压力下分辨率的测试 (为了方便计算压力将一块 $5 \mathrm{~mm} \times 9 \mathrm{~mm}$ 矩形硅片放置在传感器表面上), 压力 变化分别约为 $3 \mathrm{~Pa}, 10 \mathrm{~Pa}$ 和 $30 \mathrm{~Pa}$, 从图 $9 \mathrm{a}$ 中可以 看到, 在加载后电阻开始变化, $\Delta R / R$ 很快达到最大 值 $0.00064 / 0.002 / 0.006$, 同时随着物体的移开, $\Delta R / R$ 很快回复到原先的初始值状态。这表明该传感器的 高灵敏度和快速响应特性也适合于检测微小压力。 其超低检测限(约 $3 \mathrm{~Pa}$ )与许多文献中用于低压力的 类似 ${ }^{[20]}$, 有效拓宽了中压传感的传感器的检测极限。

将压力传感器通过聚氨酯薄膜胶带粘附在受试 者的颈部上, 让压力传感器检测人讲话时人体颈部 细微的肌肉运动。如图 $9 \mathrm{~b}$ 所示, 当测试者分别说出 不同的词语如 “Great”, “Good morning”, “Hello” 时, 压力传感器表现出非常好的敏感性和独特的波 形曲线。可以看出, 不同的发音对应于曲线中的不 同波形，且其具有很好的稳定性及对应于不同发声 产生的波形具有很好区分度, 这主要是由于在讲话 期间喉部周围的复杂肌肉运动引起的。结果表明, 基于 GNPs/SWCNT 复合材料的压力传感器可以为 语音识别提供一种简便有效的方法。

利用机械按压和手指按压来模拟中压载荷, 检 验压力传感器在中压范围内实际应用时的压敏性 能。如图 10 所示, 利用测试台或者手指按压施加测

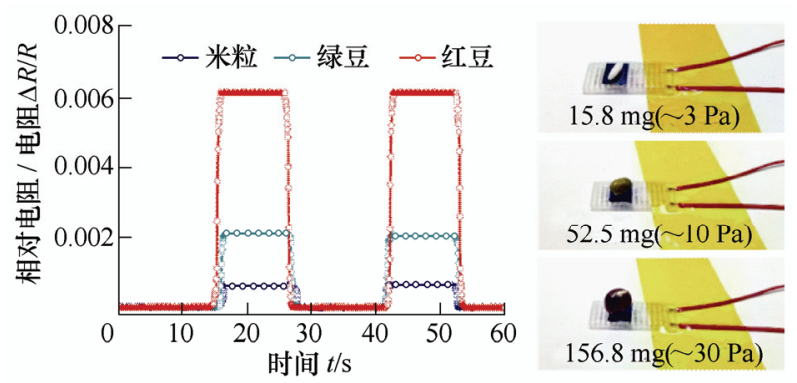

(a) 传感器分辨率测试
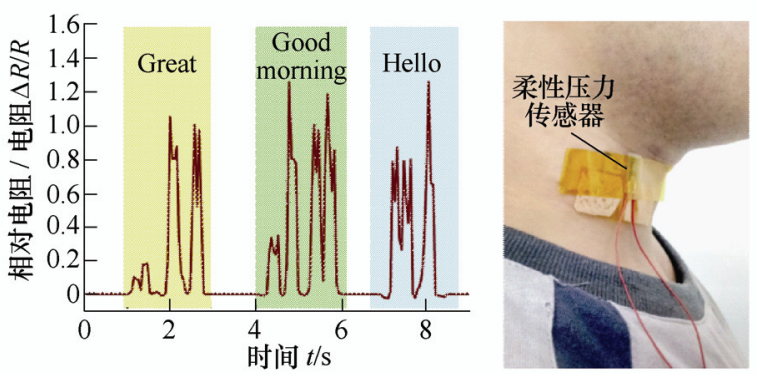

(b) 发声敏感测试

图 9 传感器分辨率及发声敏感测试图

试压力, 可以明显看出压力传感器的电阻在压力施 加时急剧上升, 从初始 $\Delta R / R$ 约为 0 上升到同一数 值或者不同数值，并在压力撤回后迅速恢复到其初 始电阻。曲线也表明压力传感器可以感知和区分不 同的压力范围。利用测试台或者手指按压施加压力 检测分为两种模式，包括相同压力的多次施加和压 力的递增或递减性施加。图 10a 示出了在测试台施 加压力情况下的响应曲线, 在施加和释放相同约 11 $\mathrm{kPa}$ 的同一压力情况下, $\Delta R / R$ 的值由初始值约为 0 增加到约为 $1, \Delta R / R$ 的最大值如预期的几乎保持在 约为 1 , 并且振幅和波形都保持了很好的一致性; 而随着压力从约 $3.5 \mathrm{kPa}$ 增加到约 $30 \mathrm{kPa}$ 再减小到 约 $4 \mathrm{kPa}, \Delta R / R$ 的最大值从约 0.23 增加到约 18 再 减小到约 0.27 。在手指按压施加测试压力的情况下, 其效果是相似的(图 10b)。从中我们可以得出结论, 压力传感器在感知触觉行为方面是可靠的, 具有高

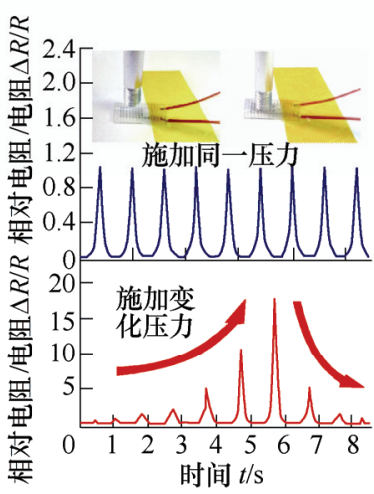

(a) 机械按压

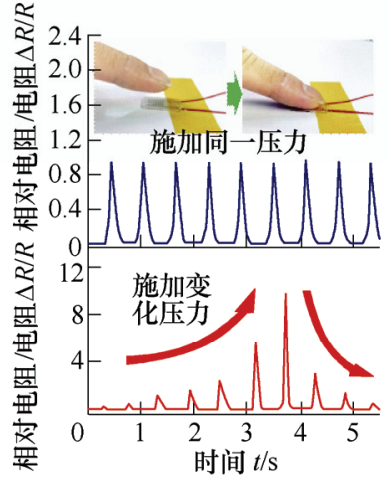

(b) 手指按压
图 10 机械按压及手指按压测试图 
灵敏度和稳定性。结合其良好压力分辨率优势, 该 压力传感器在人工假肢和康复机器人等应用中将起 到非常巨大的作用。

\section{4 结论}

(1) 本文提出在 PDMS 基材上通过直写喷印 GNPs/MWCNT 复合材料墨水形成 S 形折线压力敏 感单元方法制备了一种柔性中压压力传感器, 避免 了 GNPs/MWCNT 产生团聚。传感器 PDMS 封装层 在与敏感单元直接接触表面构建微小线条结构阵 列, 有效提高了压力传感器的灵敏度和分辨率。

(2) 测试结果表明, 在 $0 \sim 15 \mathrm{kPa}$ 及 $15 \sim 40 \mathrm{kPa}$ 压力下, 传感器灵敏度分别为 $0.114 \mathrm{kPa}^{-1}$ 和 1.41 $\mathrm{kPa}^{-1}$ 。同时, 其可检测低至 $3 \mathrm{~Pa}$ 的微小压力变化, 有效拓宽了中压传感器的检测限。该压力传感器展 示出快速响应/恢复特性(约 $100 \mathrm{~ms} / 50 \mathrm{~ms}$ )、良好重 复性和循环稳定性。

(3) 柔性压力传感器对不同发声展现出可区分 的稳定信号及对不同指压触摸动作的精确稳定信号 反馈, 预示着它未来极有可能在语音识别、人工假 肢和康复机器人、远程医疗等实际应用中将起到重 要的作用。

\section{参 考 文 献}

[1] WANG S, XU J, WANG W, et al. Skin electronics from scalable fabrication of an intrinsically stretchable transistor array[J]. Nature, 2018, 555(7694): 83.

[2] MA Y, YUE Y, ZHANG H, et al. 3D synergistical MXene/reduced graphene oxide aerogel for a piezoresistive sensor[J]. ACS Nano, 2018, 12(4): 3209-3216.

[3] KIM J, LEE M, SHIM H J, et al. Stretchable silicon nanoribbon electronics for skin prosthesis[J]. Nature Communications, 2014, 5: 5747.

[4] GE J, SUN L, ZHANG F R, et al. A stretchable electronic fabric artificial skin with pressure-, lateral strain-, and flexion-sensitive properties[J]. Advanced materials, 2016, 28(4): 722-728.

[5] LUO N, DAI W, LI C, et al. Flexible piezoresistive sensor patch enabling ultralow power cuffless blood pressure measurement[J]. Advanced Functional Materials, 2016, 26(8): 1178-1187.

[6] KIM J, KIM M, LEE M S, et al. Wearable smart sensor systems integrated on soft contact lenses for wireless ocular diagnostics $[\mathrm{J}]$. Nature Communications, 2017, 8: 14997.
[7] BAUER S, BAUER-GOGONEA S, GRAZ I, et al. 25th anniversary article: A soft future: From robots and sensor skin to energy harvesters[J]. Advanced Materials, 2014, 26(1): 149-162.

[8] JUNG S, KIM J H, KIM J, et al. Reverse-micelle-induced porous pressure-sensitive rubber for wearable human-machine interfaces[J]. Advanced Materials, 2014, 26(28): 4825-4830.

[9] TRUNG T Q, LEE N E. Flexible and stretchable physical sensor integrated platforms for wearable human-activity monitoringand personal healthcare[J]. Advanced Materials, 2016, 28(22): 4338-4372.

[10] YEO J C, LIM C T. Emerging flexible and wearable physical sensing platforms for healthcare and biomedical applications[J]. Microsystems \& Nanoengineering, 2016, 2: 16043 .

[11] TAO L Q, ZHANG K N, TIAN H, et al. Graphene-paper pressure sensor for detecting human motions[J]. ACS nano, 2017, 11(9): 8790-8795.

[12] BOLAND C S, KHAN U, RYAN G, et al. Sensitive electromechanical sensors using viscoelastic graphene-polymer nanocomposites[J]. Science, 2016, 354(6317): 1257-1260.

[13] LEE S, REUVENY A, REEDER J, et al. A transparent bending-insensitive pressure sensor[J]. Nature Nanotechnology, 2016, 11(5): 472.

[14] PAN L, CHORTOS A, YU G, et al. An ultra-sensitive resistive pressure sensor based on hollow-sphere microstructure induced elasticity in conducting polymer film[J]. Nature Communications, 2014， 5: 3002.

[15] LOU Z, CHEN S, WANG L, et al. An ultra-sensitive and rapid response speed graphene pressure sensors for electronic skin and health monitoring $[\mathrm{J}]$. Nano Energy, 2016, 23: 7-14.

[16] CHOONG C L, SHIM M B, LEE B S, et al. Highly stretchable resistive pressure sensors using a conductive elastomeric composite on a micropyramid array[J]. Advanced Materials, 2014， 26(21): 3451-3458.

[17] PARK J, LEE Y, HONG J, et al. Tactile-directionsensitive and stretchable electronic skins based on human-skin-inspired interlocked microstructures[J]. ACS Nano, 2014, 8(12): 12020-12029.

[18] PANG C, LEE G Y, KIM T, et al. A flexible and highly sensitive strain-gauge sensor using reversible interlocking of nanofibres[J]. Nature Materials, 2012, 11(9): 795.

[19] MANNSFELD S C B, TEE B C K, STOLTENBERG R $\mathrm{M}$, et al. Highly sensitive flexible pressure sensors with microstructured rubber dielectric layers[J]. Nature Materials, 2010, 9(10): 859 . 
[20] ZHU B, NIU Z, WANG $\mathrm{H}$, et al. Microstructured graphene arrays for highly sensitive flexible tactile sensors[J]. Small, 2014, 10(18): 3625-3631.

[21] ZHU B, WANG H, LIU Y, et al. Skin-inspired haptic memory arrays with an electrically reconfigurable architecture[J]. Advanced Materials, 2016, 28(8) : 1559-1566.

[22] WANG X, GU Y, XIONG Z, et al. Silk-molded flexible, ultrasensitive, and highly stable electronic skin for monitoring human physiological signals[J]. Advanced Materials, 2014, 26(9): 1336-1342.

[23] HARADA S, KANAO K, YAMAMOTO Y, et al. Fully printed flexible fingerprint-like three-axis tactile and slip force and temperature sensors for artificial skin[J]. ACS Nano, 2014, 8(12): 12851-12857.

[24] RYU S, LEE P, CHOU J B, et al. Extremely elastic wearable carbon nanotube fiber strain sensor for monitoring of human motion[J]. ACS Nano，2015，9(6): 5929-5936.

[25] LIANG J , TONG K, PEI Q. A water-based silver-nanowire screen-print ink for the fabrication of stretchable conductors and wearable thin-film transistors[J]. Advanced Materials, 2016, 28(28) : 5986-5996.

[26] ZHOU J, ELLIS A V, VOELCKER N H. Recent developments in PDMS surface modification for microfluidic devices[J]. Electrophoresis, 2010, 31(1): 2-16.

[27] PANG Y, ZHANG K, YANG Z, et al. Epidermis microstructure inspired graphene pressure sensor with random distributed spinosum for high sensitivity and large linearity[J]. ACS Nano, 2018, 12(3): 2346-2354.

[28] TIAN H, SHU Y, WANG X F, et al. A graphene-based resistive pressure sensor with record-high sensitivity in a wide pressure range[J]. Scientific Reports, 2015, 5: 8603.

[29] FERRARI A C, ROBERTSON J. Resonant Raman spectroscopy of disordered, amorphous, and diamondlike carbon[J]. Physical Review B Condensed Matter, 2002, 64(7): 075414.

[30] MOHIUDDIN T M G, LOMBARDO A, NAIR R R, et al. Uniaxial strain in graphene by Raman spectroscopy: $G$ peak splitting, Gruneisen parameters, and sample orientation[J]. Physical Review B. 2008, 79(20): 205433.

[31] HWANG S H, PARK H W, PARK Y B. Piezoresistive behavior and multi-directional strain sensing ability of carbon nanotube-graphene nanoplatelet hybrid sheets[J]. Smart Materials and Structures, 2012，22(1): 015013.

[32] PARK H, JEONG Y R, YUN J, et al. Stretchable array of highly sensitive pressure sensors consisting of polyaniline nanofibers and Au-coated polydimethylsiloxane micropillars[J]. ACS Nano, 2015，9(10): 9974-9985.

作者简介: 罗毅辉, 男, 1993 年出生。主要研究方向为电子皮肤。 E-mail: 19920161151457@stu.xmu.edu.cn 吴德志(通信作者), 男, 1977 年出生, 博士, 教授, 博士研究生导师。 主要研究方向为微纳制造、柔性电子及应用。

E-mail:wdz@xmu.edu.cn

朱睿(通信作者), 男, 1980 年出生, 博士, 助理教授。主要研究方向为 流体力学。

E-mail: zhurui@xmu.edu.cn 